\title{
Optimization of Top Coupling Grating for Very Long Wavelength QWIP Based on Surface Plasmon
}

\author{
Guodong WANG ${ }^{1 *}$, Junling SHEN ${ }^{2}$, Xiaolian $\mathrm{LIU}^{1}, \mathrm{Lu} \mathrm{NI}^{2}$, and Saili WANG ${ }^{2}$ \\ ${ }^{1}$ School of Physics and Electronic Information Engineering, Henan Polytechnic University, No.2001, Shiji road, Jiaozuo, \\ 454003, China \\ ${ }^{2}$ School of Electrical Engineering and Automation, Henan Polytechnic University, No.2001, Shiji road, Jiaozuo, 454003, \\ China \\ *Corresponding author: Guodong WANGＩ-mail: wgd@hpu.edu.cn
}

\begin{abstract}
The relative coupling efficiency of two-dimensional (2D) grating based on surface plasmon for very long wavelength quantum well infrared detector is analyzed by using the three-dimensional finite-difference time domain (3D-FDTD) method algorithm. The relative coupling efficiency with respect to the grating parameters, such as grating pitch, duty ratio, and grating thickness, is analyzed. The calculated results show that the relative coupling efficiency would reach the largest value for the $14.5 \mu \mathrm{m}$ incident infrared light when taking the grating pitch as $4.4 \mu \mathrm{m}$, the duty ratio as 0.325 , and the grating thickness as $0.07 \mu \mathrm{m}$, respectively.
\end{abstract}

Keywords: Very long wavelength; QWIP; surface plasmon; 2D grating

Citation: Guodong WANG, Junling SHEN, Xiaolian LIU, Lu NI, and Saili WANG, "Optimization of Top Coupling Grating for Very Long Wavelength QWIP Based on Surface Plasmon,” Photonic Sensors, 2017, 7(3): 278-282.

\section{Introduction}

The very long wavelength infrared (VLWIR) spectral range from $14 \mu \mathrm{m}$ to $20 \mu \mathrm{m}$ is of great importance for space applications [1,2]. Quantum well infrared photodetectors (QWIPs) based on the $\mathrm{GaAs} / \mathrm{AlGaAs}$ material system have been studied in detail and have already been used in large format focal plane arrays [3-6]. Because of its mature growth and processing technology, QWIPs have been investigated as an alternative for VLWIR detection $[7,8]$.

However, VLWIR QWIPs have a lower performance than that of $\mathrm{HgCdTe}$ detectors and suffer from low response photocurrent, partly because of the lower coupling efficiency of top metal grating. Therefore, it is quite necessary to improve the performances of VLWIR QWIPs.

Since Ebbesen [9] discovered the interesting extraordinary optical transmission of periodic holes array perforated in the metal film, the surface plasmon (SP) has attracted a lot of research interests. SP has been applied in different kinds of devices, such as semiconductors lasers, quantum dot solar cells, and infrared detectors [10-12]. In the very long wavelength infrared band, the SP propagation length is large enough to effectively reach the entire quantum well region, and the optical loss is relatively small. So if SP is applied in VLWIR QWIP, the coupling efficiency of grating and

Received: 18 May 2017 / Revised: 11 June 2017

(C) The Author(s) 2017. This article is published with open access at Springerlink.com DOI: $10.1007 / \mathrm{s} 13320-017-0433-1$

Article type: Regular 
response photocurrent will be improved evidently.

In this paper, the coupling grating based on SP for the VLWIR QWIPs is simulated by the finite-difference time domain method (FDTD). The relative coupling efficiencies with respect to different grating parameters are compared, and the optimal parameters of grating have been obtained.

\section{Simulation model}

The three-dimensional finite-difference time domain method (3D-FDTD) is strictly a numerical algorithm for solving Maxwell's equations. It can be applied to simulate the SP waves and electric field component distribution. The simulated structure is shown in Fig. 1. From the bottom to top, the QWIP is composed of GaAs substrate, $n$-type GaAs bottom contact layer, GaAs/AlGaAs multiple quantum well layer, $n$-type GaAs top contact layer, and grating layer (periodic hole array). The source is a normally incident plane wave. For convenience, the growth direction of the MQW layer is set to $z$-coordinate, the bottom of grating layer is set to $z=0$, the device plane is set to $x-y$ plane, and the central point of $x-y$ plane is set to $x=0, y=0 . P$ and $D$ respectively represent the grating pitch and the diameter of the hole. Supposing the light incident from the top, the coupling efficiency of $x-y$ at $z$ point can be expressed as [13]

$$
\eta(z)=\frac{\iint E_{z}^{2}(x, y, z) d x d y}{\iint E_{\text {in }}^{2}(x, y, z) d x d y}
$$

where $E_{\text {in }}$ represents the electric field component of the incident light, and $E_{z}$ represents the electric field component along the $z$ axis in the $x-y$ plane.

One attraction is that because of the limitation of computer, all the MQW region is not selected to integration. Accordingly, a reasonable integral area is selected and fixed, so the relative coupling efficiency is obtained. This is only due to the limitations of numerical calculation, but does not affect the results of the physical characteristics of the performance. In this paper, we fix the integration area as $9.6 \mu \mathrm{m} \times 9.6 \sqrt{3} \mu \mathrm{m}$ in the center of $x-y$ plane.

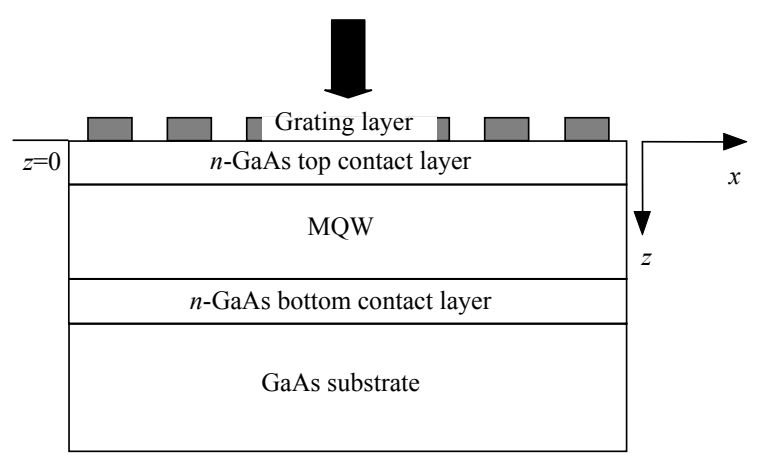

(a)

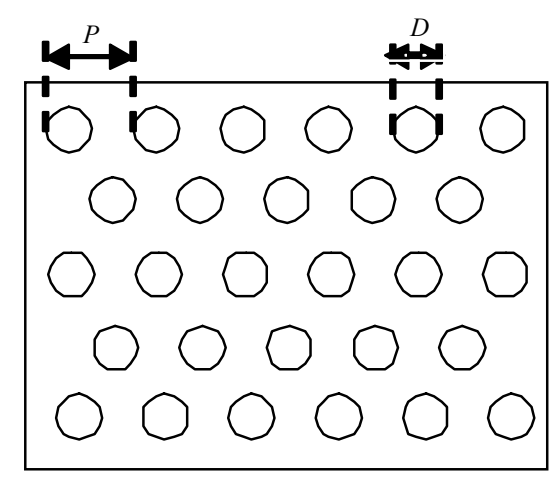

(b)

Fig. 1 Modelling structure of VLWIR QWIP: (a) device structure and coordinate direction and (b) structure of periodic hole array.

\section{Calculation and analysis}

Firstly, by setting the incident light as TE mode plane wave $\left(E_{z}=0\right)$ and the central wavelength of incident source as $14.5 \mu \mathrm{m}$, the grating parameters as grating pitch $P=2.8 \mu \mathrm{m}$, the diameter of hole $D=$ $1.6 \mu \mathrm{m}$, the thickness of grating layer $L=0.08 \mu \mathrm{m}$, and the $E_{z}$ field in $x-y$ plane are calculated by FDTD. The $E_{z}$ distribution at $z=0.11 \mu \mathrm{m}$ is illustrated in Fig. 2. As can be seen from Fig. 2, the direction of infrared light propagation has been changed obviously, and $E_{z}$ is concentrated on the position in correspondence with the grating holes.

Further calculation shows that the intensity of the light in different $x-y$ planes is decreased with an increase in $z$, which follows the exponential law, as 
shown in Fig. 3. In other words, it can be concluded that the farther away from the grating is, the lower intensity of light in $x-y$ plane is. It also fits well with the propagation properties of SP wave. So it can be proved that $E_{z}$ in the $x-y$ plane is indeed excited by SP.

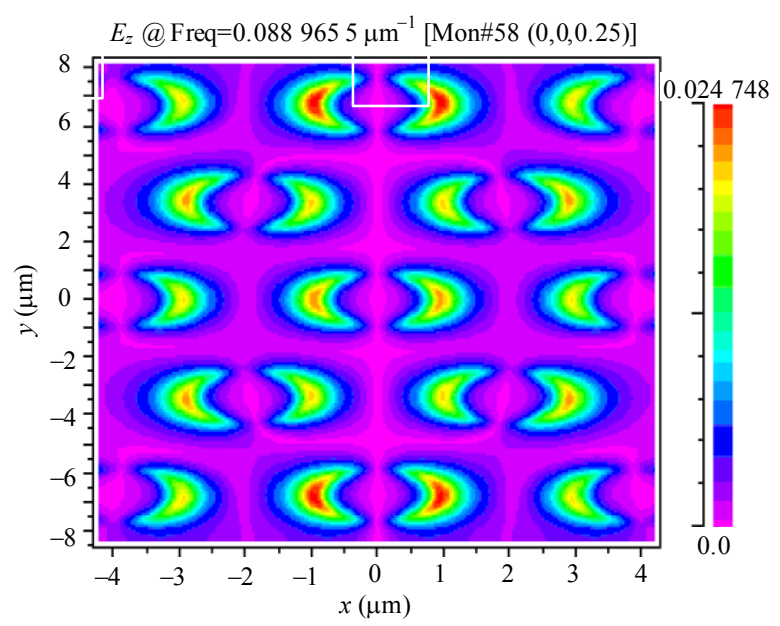

Fig. 2 Distribution of $E_{z}$ at $z=0.11 \mu \mathrm{m} x-y$ plane.

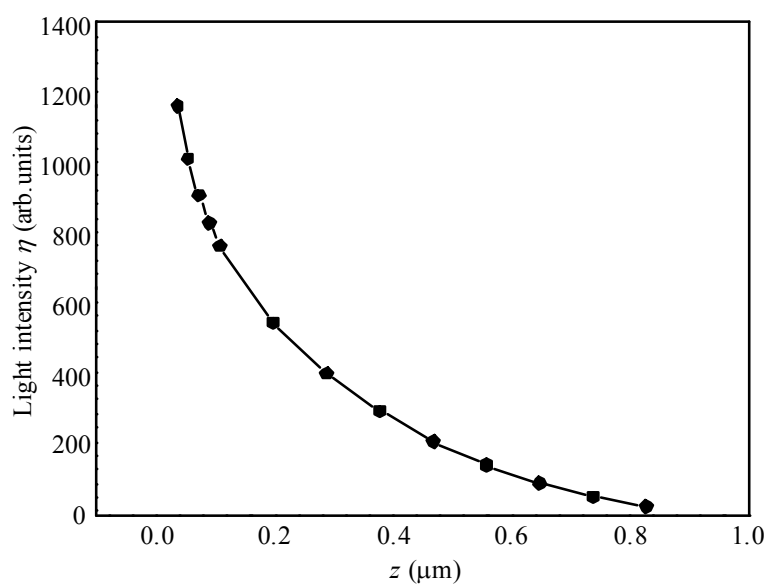

Fig. 3 Distribution of $E_{z}$ at different $x-y$ planes.

Secondly, in order to obtain the more accurate results, the relative coupling efficiency of grating is calculated by (1) in the fixed integral area which is described above. Figure 4 shows the relative coupling efficiency with respect to the grating pitch. It can be seen that the grating coupling efficiency reaches the maximum when the grating pitch is $4.4 \mu \mathrm{m}$. This result is in agreement with the grating equation.

And then, by taking the grating pitch as
$P=4.4 \mu \mathrm{m}$, the relative coupling efficiency is calculated when the duty ratio $D / P$ is changed. The calculated results are shown in Fig. 5. It shows that the peak value of relative coupling efficiency is achieved when the duty ratio is taken as 0.325 .

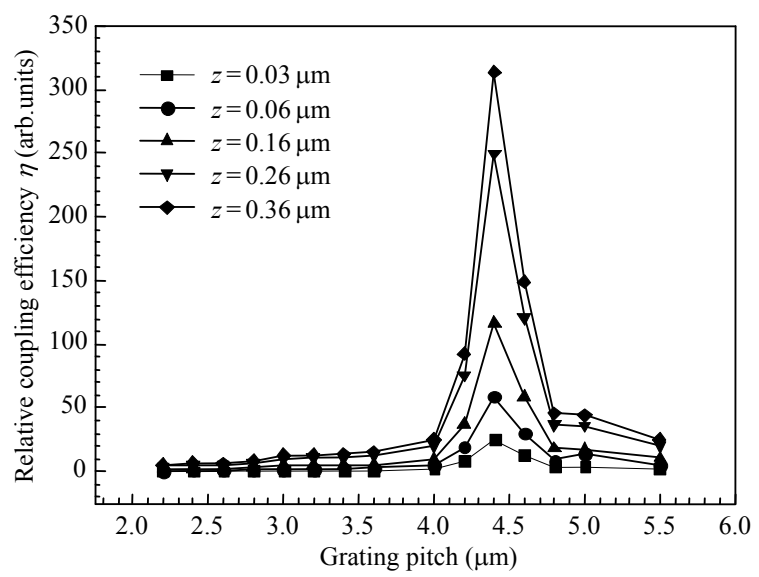

Fig. 4 Relative coupling efficiency with respect to grating pitch.

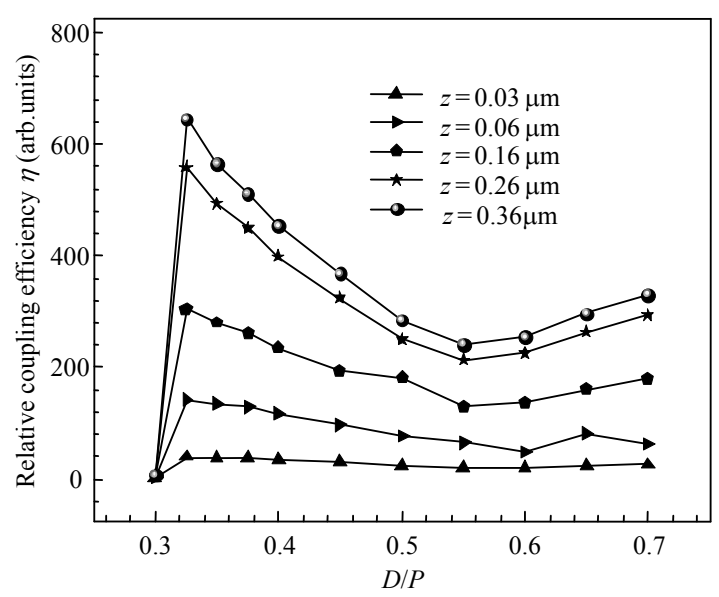

Fig. 5 Relative coupling efficiency with respect to duty ratio $D / P$.

Then, by taking duty ratio as $D / P=0.325$, the relative coupling efficiency is calculated with respect to the grating thickness. The calculated results are shown in Fig. 6. It can be concluded that the largest relative coupling efficiency will be obtained when the grating thickness is $0.07 \mu \mathrm{m}$.

Finally, the optimized parameters of coupling grating for $15.4 \mu \mathrm{m}$ input infrared light is obtained, which is illustrated as the grating pitch $4.4 \mu \mathrm{m}$, the duty ratio 0.325 , and the grating thickness $0.07 \mu \mathrm{m}$. 


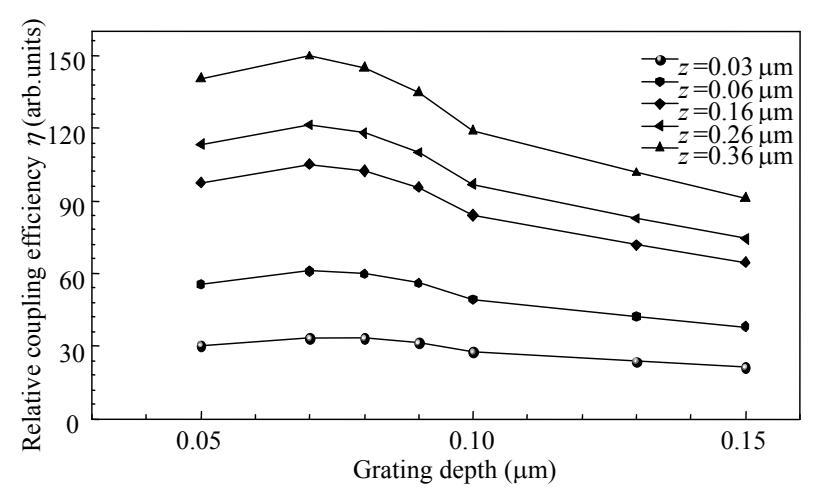

Fig. 6 Relative coupling efficiency with respect to grating thickness.

\section{Conclusions}

In conclusion, the relative coupling efficiency of top 2D metal coupling grating based on SP for the VLWIR QWIP is calculated by FDTD. The optimized parameters grating is obtained by maximizing the coupling efficiency for different grating parameters. This work is beneficial to enhancing the performance of the very long wavelength QWIP focal plane array devices.

\section{Acknowledgement}

This work was supported by the National Natural Science Foundation of China under Grant No. U1304608, the Outstanding Youth Funding of Henan Polytechnic University under Grant No. J2013-05, and Program for Innovative Research Team of Henan Polytechnic University under Grant No. T2015-3.

Open Access This article is distributed under the terms of the Creative Commons Attribution 4.0 International License (http://creativecommons.org/ licenses/by/4.0/), which permits unrestricted use, distribution, and reproduction in any medium, provided you give appropriate credit to the original author(s) and the source, provide a link to the Creative Commons license, and indicate if changes were made.

\section{References}

[1] Y. Wei, W. Q. Ma, Y. H. Zhang, J. L. Huang, Y. L. Cao, and K. Cui, "High structural quality of type II
InAs/GaSb superlattices for very long wavelength infrared detection by interface control," IEEE Journal of Quantum Electronics, 2012, 48(4): 512-515.

[2] A. M. Hoang, G. Chen, R. Chevallier, A. Haddadi, and M. Razeghi, "High performance photodiodes based on InAs/InAsSb type-II superlattices for very long wavelength infrared detection," Applied Physics Letters, 2014, 104(25): 251105-1-251105-4.

[3] A. Rogalski, "Recent progress in infrared detector technologies," Infrared Physics \& Technology, 2011, 54(3): 136-154.

[4] S. D. Gunapala, D. Z. Ting, C. J. Hill, J. Nguyen, A. Soibel, S. B. Rafol, et al., "Large area III-V infrared focal planes," Infrared Physics \& Technology, 2011, 54(3): 155-163.

[5] G. D. Wang, S. L. Dai, and H. Zhang, "Optimization fo top coupling grating for mid-wave quantum well infrared photodetector," Chinese Optics Letters, 2012, 10(B06): 188-189.

[6] W. Lu, L. Li, H. L. Zheng, W. L. Xu, and D. Y. Xiong, "Development of an infrared detector: quantum well infrared photodetector," Science China Physics, Mechanics \& Astronomy, 2009, 52(7): 969-977.

[7] X. H. Liu, X. H. Zhou, N. Li, L. Wang, Q. L. Sun, K. S. Liao, et al., "Effects of bias and temperature on the intersubband absorption in very long wavelength GaAs/AlGaAs quantum well infrared photodetectors," Journal of Applied Physics, 2014, 115(12): 124503.

[8] A. Berurier, A. Nedelcu, V. Gueriaux, T. Bria, A. D. Rossi, X. Marcadet, et al., "Optimization of broadband $(11-15 \mu \mathrm{m})$ optical coupling in quantum well infrared photodetectors for space applications," Infrared Physics \& Technology, 2011, 54(3): 182-188.

[9] C. Genet and T. W. Ebbesen, "Light in tiny holes," Nature, 445(7123): 39-46.

[10] W. Wu, A. Bonakdar, and H. Mohseni, "Plasmonic enhanced quantum well infrared photodetector with high detectivity," Applied Physics Letters, 2010, 96(16): 161107-1-161107-3.

[11] C. C. Chang, Y. D. Sharma, Y. S. Kim, J. A. Bur, R. V. Shenoi, S. Krishna, et al., "A surface plasmon enhanced infrared photodetector based on InAs quantum dots," Nano Letters, 2010, 10(5): 1704-1709.

[12] Q. C. Weng, L. Li, J. Chen, J. Wen, and D. Y Xiong, "The metal grating coupling of long wavelength quantum well infrared photodetectors: surface 
plasmon effect," Journal of Infrared \& Millimeter Waves, 2011, 30(5): 415-418.

[13] K. Wang, W. H. Zheng, G. Ren, X. Y. Du, M. X. Xing, and L. H. Chen, "Design and optimization color quantum well infrared photodetectors coupled photonic crystal layer," Acta Physica Sinica, 2008, 57(3): 1730-1735. 